\title{
Hierarchical Two-Dimensional Layered Double Hydroxide Coated Polydopamine Nanocarriers for Combined Chemodynamic and Photothermal Tumor Therapy
}

\author{
Prabhakar Busa ${ }^{1} \mathbb{D}$, Ravindranadh Koutavarapu $\left.{ }^{2, *} \mathbb{(}\right)$, Dong-Yeon Lee ${ }^{2, *}$, Jaesool Shim ${ }^{3, *}$ and Yaswanth Kuthati ${ }^{1, *}$ \\ 1 Department of Anesthesiology, Cathay General Hospital, Taipei 280, Taiwan; prabhakar.busa01@gmail.com \\ 2 Department of Robotics and Intelligent Machine Engineering, College of Mechanical and IT Engineering, \\ Yeungnam University, Gyeongsan 712-749, Korea \\ 3 School of Mechanical Engineering, Yeungnam University, Gyeongsan 712-749, Korea \\ * Correspondence: ravindra_physicist@ynu.ac.kr (R.K.); dylee@ynu.ac.kr (D.-Y.L.); jshim@ynu.ac.kr (J.S.); \\ yaswanthk1987@gmail.com (Y.K.)
}

Citation: Busa, P.; Koutavarapu, R.; Lee, D.-Y.; Shim, J.; Kuthati, Y. Hierarchical Two-Dimensional Layered Double Hydroxide Coated Polydopamine Nanocarriers for Combined Chemodynamic and Photothermal Tumor Therapy.

Coatings 2021, 11, 1008.

https://doi.org/10.3390/coatings 11081008

Received: 19 July 2021

Accepted: 19 August 2021

Published: 23 August 2021

Publisher's Note: MDPI stays neutral with regard to jurisdictional claims in published maps and institutional affiliations.

Copyright: (c) 2021 by the authors. Licensee MDPI, Basel, Switzerland. This article is an open access article distributed under the terms and conditions of the Creative Commons Attribution (CC BY) license (https:/ / creativecommons.org/licenses/by/ $4.0 /)$.

\begin{abstract}
The combination of chemodynamic therapy (CDT) and photothermal therapy (PTT) has proven to be successful in combating the challenges associated with cancer therapy. A combination of these therapies can maximize the benefits of each therapeutic modality through endogenous reduction-oxidation (redox) reaction and external laser power induction. In the current work, we have designed a copper-aluminum layered double hydroxide (CuAl-LDH) loaded doxorubicin (DOX) by a co-precipitation method; the surface was coated with polydopamine (PDA). The synthesized CuAlLDH@DOX@PDA nanocarrier (NC) served as a Fenton-like catalyst with photothermal properties. It is well known that metal ion incorporated NCs can induce intracellular depletion of reduced glutathione (GSH) levels along with the reduction of $\mathrm{Cu}^{2+}$ to $\mathrm{Cu}^{+}$. The $\mathrm{Cu}^{+}$ions in turn react with DOX leading to the generation of intracellular hydrogen peroxide $\left(\mathrm{H}_{2} \mathrm{O}_{2}\right)$ molecules to produce the highly toxic hydroxyl radicals $(\bullet \mathrm{OH})$ through a Fenton-like reaction. The enhanced absorption of CuAl@DOX@PDA at 810 nm, greatly improved the photothermal efficiency in comparison with bare CuAl-LDH and CuAl-LDH@DOX. In vitro studies revealed the tremendous CDT/PTT efficacy of CuAl@DOX@PDA in suppressing A549 cancer cells. Furthermore, reactive oxygen species (ROS) assays and intracellular levels of various ROS cascade biomolecules support our findings in the efficient destruction of cancer cells through synergistic CDT/PTT therapy.
\end{abstract}

Keywords: layered double hydroxide (LDH); chemodynamic therapy (CDT); photothermal therapy (PTT); doxorubicin (DOX); polydopamine (PDA); Fenton-like reaction; reactive oxygen species (ROS); cancer therapy

\section{Introduction}

Cancer is the leading cause of death worldwide [1]. Several approaches have been developed for the management of cancers, including chemotherapy (CT), CDT, PTT, photodynamic therapy (PDT), photothermal therapy, radiotherapy (RT), and surgical therapy [2]. However, the use of a single therapeutic modality for cancer treatment is often associated with limitations such as minimal therapeutic efficacy, toxicity, reoccurrence of the malignancy, and resistance to a particular type of treatment [3]. To overcome these limitations associated with monotherapy, synergistic treatment modalities combining different treatments have evolved and have proven to be advantageous [4]. In recent years, the synergistic effects of CDT and PTT have been proven to be beneficial in the suppression of cancers [5,6]. NC-based therapeutic delivery has gained widespread attention recently due to the benefits such as site-specific drug delivery, targeted drug delivery, bio-imaging, cancer therapy, and applications in other bio-logical fields [7-9]. Various types of NC were used for biological applications such as mesoporous silica, polymeric NC, carbon 
nanotubes, lipid-based NCs, and LDH [10]. Among the NCs studied, LDH has versatile characteristics, such as adjustable elemental composition, good biocompatibility, and high interlayer gallery space facilitating a high drug loading capacity. Previous in vivo studies have confirmed that LDH NCs are non-toxic to animals without any signs of inflammation, blood obstruction, and histological changes after four weeks of treatment [11-14].

In our current report, the intercalation of the chemotherapeutic drug in the LDH interlayers is efficient in ROS generation inside the cells through cellular pathways, whereas the transitional metal in the layered architecture can generate highly toxic hydroxyl radicals through a Fenton-like reaction by CDT. In addition, PDA aids in PTT through synergistic enhancement of $\mathrm{CT}$ and CDT effects by increasing cell death via heat generation from the specific light source. Lu et al. reported dihydroartemisinin-loaded manganese ion-doped magnesium-Iron-LDH NCs for synergistic CDT/PTT [15]. The developed NC's showed tremendous photothermal properties with a photothermal conversion efficacy of up to $10.7 \%$ [15]. In another study, Sun et al. reported indocyanine green intercalated copper $\mathrm{CCu}$ substituted LDH for combinational PTT/PDT therapy to inhibit tumor growth in vivo. The intercalated metal ion is shown to generate a Fenton-like reaction with amplification of ROS species through the $\mathrm{Cu}^{+}$oxidation [16]. Several other researchers have developed hybrid LDH NC's by incorporation of various metal ions for multimodal synergistic therapies [17-21].

In our current work, we reported CuAl-LDH/ICG NC's for tri-modal cancer therapy viz, CDT/PDT/PTT. Initially, we synthesized CuAl-LDH through the co-precipitation method and further intercalated the chemotherapeutic DOX within the LDH interlayers. Further PDA is coated over the surface to aid in PTT and sustained drug release. The in-vitro cell viability, cellular uptake studies, GSH depletion, and ROS detection assays prove that the synthesized NCs are efficient in inhibiting cancer cell proliferation. The synergistic mode of action of our NCs is explained in the scheme.

\section{Materials and Methods}

\subsection{Reagents}

Aluminum chloride hexahydrate $\left(\mathrm{AlCl}_{3} \cdot 6 \mathrm{H}_{2} \mathrm{O}\right)$ and Sodium hydroxide $(\mathrm{NaOH})$ were purchased from J.T. Bakers (Clare, MI, USA). Copper chloride dihydrate $\left(\mathrm{CuCl}_{2} \cdot 2 \mathrm{H}_{2} \mathrm{O}\right)$, Doxorubicin hydrochloride (DOX·HCl), 2,7-Dichlorofluorescin diacetate (DCFDA), and Dopamine were purchased from the Sigma Aldrich (St. Louis, MO, USA).

\subsection{Characterization}

The transmission electron microscopic (TEM) images of LDH were captured by a transmission electron microscope Hitachi HT7700, (Tokyo, Japan) ran at an acceleration voltage of $120 \mathrm{kV}$. X-ray powder diffraction (XRD) analysis was conducted by X-ray diffractometer (Bruker, Robin Hill Road, Santa Barbara, CAy; Cu K $\alpha$ radiation, $\Lambda=0.15405 \mathrm{~nm}$ ) at $30 \mathrm{kV}$ and $30 \mathrm{~mA}$ with a scanning rate of $10^{\circ} / \mathrm{min}$ with $2 \theta$ ranging from $10^{\circ}$ to $80^{\circ}$. The zeta size and zeta potential were obtained by using Zetasizer Nano ZS-90, (Malvern, UK). The cell viability assay was determined by using a PerkinElmer Enspire Multimode Plate Reader (Waltham, MA, USA). The functional group analysis was done by Fourier transmission infrared spectroscopic (FTIR) studies, which were recorded on Brucker's Tensors 27 series spectrometer (Texas city, TX, USA). Ultraviolet-visible (UV-Vis) absorbance studies were carried out by Uv-1700 Pharma Spec, Shimadzu, MA (canton), USA.

\subsection{Synthesis of $L D H$}

LDH NCs were prepared by co-precipitation method by following the previously reported methods [22]. Briefly, $1.5 \mathrm{mmol} \mathrm{CuCl} 2 \cdot 2 \mathrm{H}_{2} \mathrm{O}$ and $0.5 \mathrm{mmol}$ of $\mathrm{AlCl}_{3} \cdot 6 \mathrm{H}_{2} \mathrm{O}$ were added into $1 \mathrm{M} \mathrm{NaOH}(40 \mathrm{~mL})$ and stirred at room temperature for $3 \mathrm{~h}$. Furthermore, the slurry $\mathrm{pH}$ was adjusted with $\mathrm{NaOH}(0.2 \mathrm{M})$ solution to 10 . The slight blue color precipitate was collected and centrifuged at 12,000 rpm for $17 \mathrm{~min}$ and the contents were washed with deionized water (degassed water) twice and the resultant solid was dispersed into $100 \mathrm{~mL}$ 
deionized water in a stainless-steel hydrothermal flask. The hydrothermal flask was placed in the oven at $100{ }^{\circ} \mathrm{C}$ for $24 \mathrm{~h}$. The solids were collected and redispersed into $30 \mathrm{~mL}$ of deionized water twice. The resultant solids were dried by a freeze-dryer and stored at $4{ }^{\circ} \mathrm{C}$ for further experiments. The product is labeled as CuAl-LDH.

\subsection{DOX Loading into Interlayers of CuAl-LDH}

The DOX was loaded into NC by following the previously reported protocols. Aqueous DOX $(1 \mathrm{mg} / \mathrm{mL})$ solution $10 \mathrm{~mL}$ was added to $200 \mathrm{mg}$ of dried CuAl-LDH and continued to stir overnight at room temperature (RT). The DOX-loaded NC was collected by centrifugation and wash with deionized water twice. The DOX loading percentage was calculated by the following equation and the product was named CuAl-LDH@DOX.

Entrapment Efficiency\% $(E E \%)=($ Amount of drug in NC/Amount of drug used in formulation $) \times 100$

Loading Efficiency\% $($ LE\% $)=($ Amount of drug in NC/Amount of NC) $\times 100$

\subsection{Surface Coating with $P D A$}

The DOX-loaded CuAl-LDH (500 mg) was dispersed into $400 \mathrm{~mL}$ of dopaminedeionized $(0.4 \mathrm{M})$ water solution. The contents were stirred at room temperature for $30 \mathrm{~min}$, and $5 \mathrm{~mL}$ of Tris- $\mathrm{HCl}$ was added to the above suspension dropwise and stirred for $24 \mathrm{~h}$. The formation of black color indicates the successful polymerization of polydopamine over CuAl-LDH@DOX NC's. Finally, PDA-coated CuAl-LDH@DOX solids were collected by centrifugation and washed several times with deionized water (degassed). The dried solid was collected by freeze-drying process and the solid was stored at $4{ }^{\circ} \mathrm{C}$ for further use. The product was labeled as CuAl-LDH@DOX@PDA.

\subsection{DOX Release Quantification}

The drug release was investigated by following the previous protocols. The percentage of DOX release was determined by immersing CuAl-LDH@DOX, and CuAlLDH@DOX@PDA in phosphate buffer solution (PBS) at two different pH (pH 5.0 and 7.4). The solids were suspended in $1 \mathrm{~mL}$ Eppendorfs at $37^{\circ} \mathrm{C}$ on a rotation apparatus at $200 \mathrm{rpm}$, and at multiple time intervals (viz 0, 1, 2, 3, 4, 5, 6, 8, 10, 14, 20, and $24 \mathrm{~h}$ ) the buffer was replaced with a $1.0 \mathrm{~mL}$ solution of fresh PBS. The amount of DOX was estimated by using UV-Vis spectroscopy by measuring DOX absorbance at $481 \mathrm{~nm}$. The experiments were performed in triplicates.

\subsection{PTT In-Vitro Studies}

The PTT studies were conducted by following previous studies, in which $1 \mathrm{~mL}$ quartz cuvette having samples were irradiated with a light source (NIR $808 \mathrm{~nm}, 1 \mathrm{~W} / \mathrm{cm}^{2}$ ) and temperature changes were recorded by the thermal camera (FLIR ONE PRO, Wilsonville, OR, USA).

\subsection{Cell Culture}

A549 cells were used for all in vitro experiments, which were cultured with Dulbecco's modified Eagle's medium (DMEM) along with 10\% FBS. 1\% penicillin-streptomycin was used as the cell culture medium, and the 3-(4.5-dimethyl-2-thiazolyl)-2,5-diphenyl tetrazolium bromide (MTT) assay was performed to measure the cell viability in various treatment groups.

\subsection{Cell Viability MTT Assay}

To evaluate the CDT efficacy in vitro, A549 cells were cultured in 96-well plates and incubated for $24 \mathrm{~h}$. The next day plates were treated with various concentrations of NC. Then, the plates were incubated for another $24 \mathrm{~h}$ and, finally, cell viability was measured by MTT colorimetric assay. To investigate the PTT efficacy in vitro, A549 cells were seeded in 96 well plates and after incubation for $24 \mathrm{~h}$; each well was treated with 
different concentrations of PDA coated NCs. After incubating for another $24 \mathrm{~h}$, each well was washed with PBS two times, and the plates were irradiated with $810 \mathrm{~nm}$ laser $(1.0 \mathrm{~W} / \mathrm{cm})$ for $5 \mathrm{~min}$ and MTT assay was performed to measure the cell viability. Formazan crystals were dissolved in DMSO.

\subsection{Invitro ROS Detection Assay}

To examine the generation of intracellular ROS, A549 cells were cultured in six-well plates and incubated for one day. Then, wells are treated with $100 \mu \mathrm{g} / \mathrm{mL}$ of CuAlLDH@DOX, and CuAl-LDH@DOX@PDA (except control wells) and PTT experiments, were carried out following the same experimental conditions by with and without using the light source. After incubation for $24 \mathrm{~h}$, every well was treated with $5 \mu \mathrm{g} / \mathrm{mL}$ DCFDA and incubated for 30 min in Corning ${ }^{\circledR}$ (Glendale, AZ, USA) 96-well plates with a transparent bottom and black sidewalls. The wells were washed with PBS solution. The generation of ROS was estimated by a microplate reader to detect the fluorescence intensity by fluorescence microscope. The experiments were performed in triplicates and hydrogen peroxide $\left(\mathrm{H}_{2} \mathrm{O}_{2}\right)$ was used as the negative control.

\subsection{GSH Levels Detection Assay}

The levels of GSH were estimated by GSH assay kits (Promega, Madison WI, USA). A549 cells were seeded onto 96 well transparent Corning ${ }^{\circledR}$ 96-well plates and treated with $\mathrm{NC}(100 \mu \mathrm{g} / \mathrm{mL})$, with and without light irradiation. After treatment, the amount of GSH levels at the cellular level were determined by following the manufacturer's instructions. The experiments were performed in triplicates. Bicinchonic acid assay (BCA) was used to measure the protein contents.

\subsection{Statistical Analysis}

All data were stated as mean \pm standard deviation (S.D.). In all analyses, $p \leq 0.001$ $\left.{ }^{* * *}\right), p \leq 0.01\left(^{* *}\right)$, and $p \leq 0.05\left(^{*}\right)$ were judged statistically significant. The data were analyzed by Student's t-test to compare two mean values and one-way ANOVA followed by Tukey's multi comparison test which was used to compare more than two mean values. Graphpad prism 5 was used to calculate the statistical significances.

\section{Results and Discussion}

\subsection{Synthesis and Anticancer Mechanism}

The rational design and synthesis of CuAl-LDH NC's are illustrated in Scheme 1. A simple co-precipitation technique was employed to produce CuAl-LDH by dispensing $\mathrm{NaOH}$ into an aqueous solution of $\mathrm{CuCl}_{2}$ and $\mathrm{AlCl}_{3}$ salts at room temperature under continuous stirring. After $3 \mathrm{~h}$, the contents were collected by centrifugation and repeated washing with deionized water thrice and aged in a hydrothermal flask for $24 \mathrm{~h}$. The collected washed, and freeze-dried NC was stored at $4{ }^{\circ} \mathrm{C}$ for further loading of DOX $\&$ PDA coating. The designed NC's display therapeutic properties by responding to the acidic conditions present in the tumor microenvironment resultant from a high glycolytic rate [23]. Most notably, $\mathrm{CuAl}-\mathrm{LDH}$ initiates CDT by the conversion of $\mathrm{Cu}$ to $\mathrm{Cu}^{2+}$ in the presence of acidic conditions of the tumor. The resultant $\mathrm{Cu}^{2+}$ could convert endogenous $\mathrm{H}_{2} \mathrm{O}_{2}$ to $\mathrm{O}_{2}$ resulting in the generation of $\bullet \mathrm{OH}$ through Fenton reaction to initiate CDT [24]. Simultaneously, the acidic environment facilitates the release of copper coordinated DOX from the LDH interlayers resulting in CDT. In addition, the polymer coating provides an extended release of DOX along with PTT upon light irradiation. 


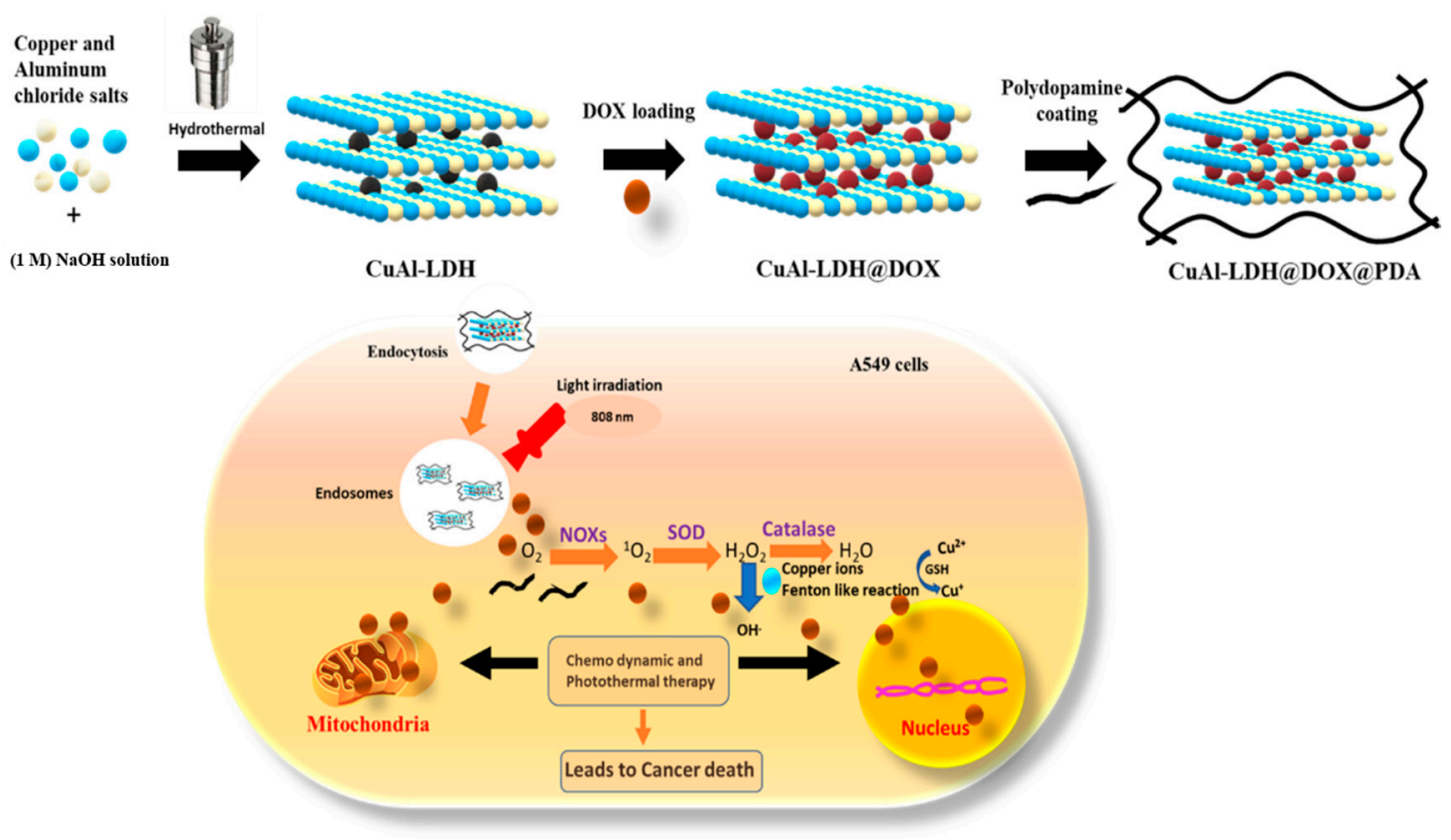

Scheme 1. Graphical representation of the synthesis of CuAl-LDH, DOX loading, and PDA coating. Cellular uptake mechanism, DOX releasing mechanism, and tumor cell eradication by chemo dynamic and photothermal therapy.

\subsection{Characterization and Properties}

Figure 1A shows the TEM image of CuAl-LDH NC's, possessing hexagonal morphology with an average size of $70 \mathrm{~nm}$. From the dynamic light scattering (DLS) studies it is evident that the mean particle size slightly increased from $70 \mathrm{~nm}$ to $90 \mathrm{~nm}$ after DOX loading (Figure $1 \mathrm{~B}(\mathrm{a}, \mathrm{b})$ ). Furthermore, PDA polymer coating resulted in a significant increase in particle size to $125 \mathrm{~nm}$ (Figure 1B(c)). Moreover, the charge of the NCs was evaluated by zeta potential measurements at $\mathrm{pH}$ 7.4. The zeta potential was positive (+35.02 and $34.23 \mathrm{mV}$ ) for both CuAl-LDH and CuAl-LDH@DOX. PDA coating slightly reduced the positive surface charge to $-14.61 \mathrm{mV}$ in CuAl-LDH@DOX@PDA confirming the polymer adsorbed to the surface of CuAl-LDH@DOX NC's. The size of LDH NCs is beneficial for cell uptake and drug release at the desired site in cancer cells, the optimal size around $100 \mathrm{~nm}$-sized NC showing promising cellular uptake by endocytosis according to the previous reports. The size and charge could influence the NC uptake, translocation efficacy into the cytosol, and drug release at the desired site of action $[8,25]$.

The FTIR spectra of the CuAl-LDH, CuAl-LDH@DOX, and CuAl-LDH@DOX@PDA are presented in Figure 2A. In CuAl-LDH samples (Figure 2A(a)) the broad characteristic bands at $3500 \mathrm{~cm}^{-1}$ correspond to $\mathrm{O}-\mathrm{H}$ stretching vibrations, whereas the band at $1625 \mathrm{~cm}^{-1}$ can be ascribed to $\mathrm{O}-\mathrm{H}$ bending of the surface $\mathrm{OH}$ functional groups and water molecules. The sharp peak at $1375 \mathrm{~cm}^{-1}$ is attributed to the interlayer chloride ions. The band at $1030 \mathrm{~cm}^{-1}$ can be assigned to a C-O stretching vibration. The presence of the characteristics bands of DOX at $1106 \mathrm{~cm}^{-1}$ and $1420 \mathrm{~cm}^{-1}$ resultant from $\delta$ (CH3O-) stretching and $\delta(\mathrm{N}-\mathrm{H})$ vibration of DOX confirms the successful loading of DOX in CuAl-LDH@DOX (Figure 2A(b,c)) [26]. Furthermore, coating of PDA resulted in additional bands at 1460 and $1630 \mathrm{~cm}-1$ which corresponds to the indole groups in PDA [27,28]. Additionally, characteristic $-\mathrm{CH}_{2}$-stretching vibrations are found at $2920 \mathrm{~cm}^{-1}$ in CuAl-LDH@DOX@PDA samples confirming successful adsorption of PDA onto CuAl-LDH@DOX (Figure 2A(d)). CuAlLDH, CuAl-LDH@DOX, and CuAl-LDH@DOX@PDA NC's showed crystalline planes structures as evident from the P-XRD patterns in Figure 2B. The layered structure of CuAlLDH with symmetrical geometry displaying different crystal planes consisting of (003), (006), and (009) values, confirming hydrotalcite structure (Figure 2B(a)). There is a slight shift of the P-XRD pattern in CuAl-LDH@DOX samples, which could be attributed to the 
d-spacing changes in brucite-like structure resulting from drug intercalation into the NC's interlayers (Figure 2B(b)). [29]. PDA loading did not affect the crystal structure and shows similar values to that of CuAl-LDH and CuAl-LDH@DOX (Figure 2B(c)).

(A)

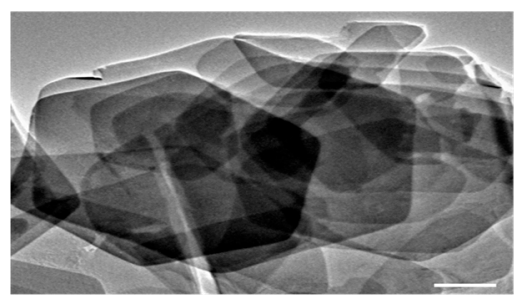

(B)

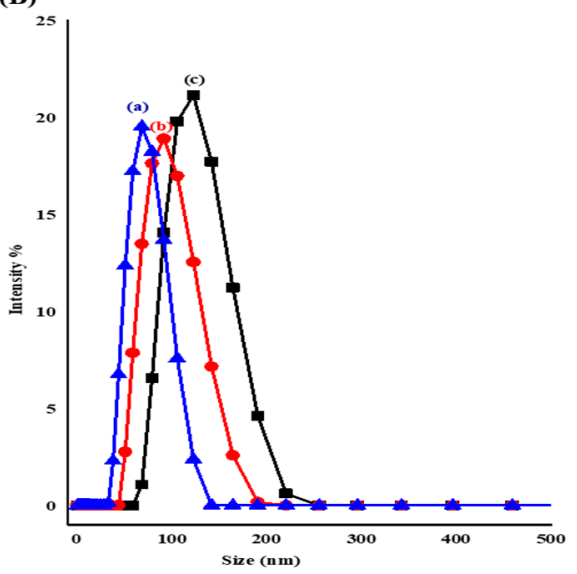

(C)

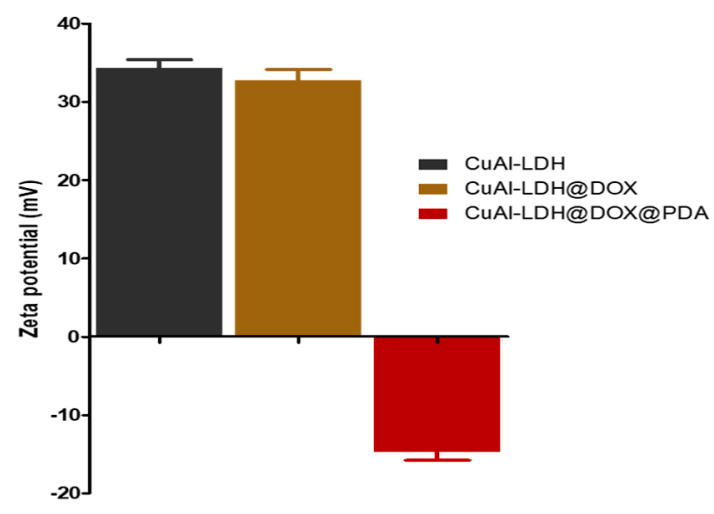

Figure 1. (A) TEM images of as-synthesized CuAl-LDH (scale bar $100 \mu \mathrm{m})$, (B) zeta size of (a) CuAl-LDH, (b) CuAl-LDH@DOX, and (c) CuAl-LDH@DOX@PDA, and (C) zeta potential of CuAl-LDH, CuAl-LDH@DOX, and CuAlLDH@DOX@PDA.

(A)

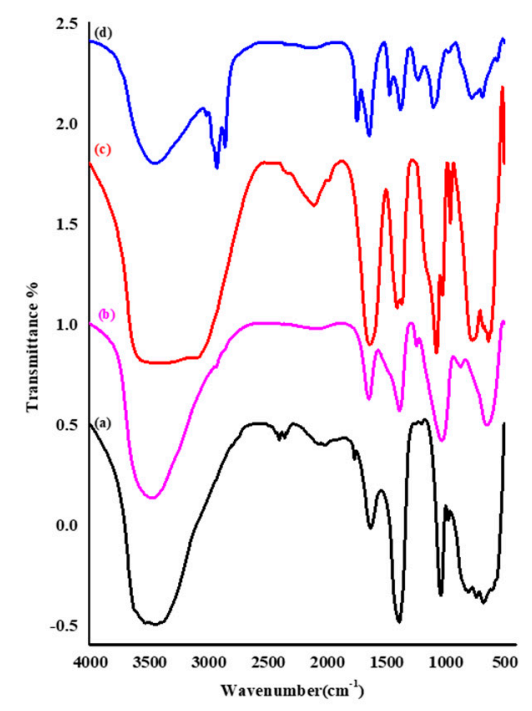

(B)

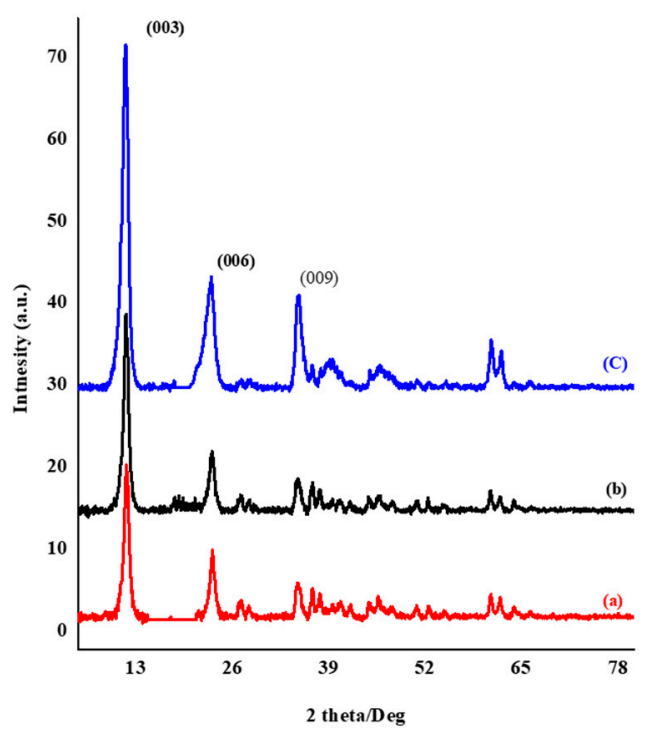

Figure 2. (A) FT-IR spectra of (a) CuAl-LDH, (b) DOX, (c) CuAl-LDH@DOX, and (d) CuAl-LDH@DOX@PDA. (B) P-XRD analysis of (a)CuAl-LDH, (b) CuAl-LDH@DOX, and (c)CuAl-LDH@DOX@PDA. 


\subsection{In Vitro DOX Release}

LDH has several desirable characteristics for drug delivery including, optimal biocompatibility, anion exchange properties, great chemical stability, and pH-dependent solubility. In particular, LDH is well known for its efficacy in delivering negatively charged therapeutics as it possesses a net positive surface charge which promotes easy adsorption and uptake through cell membranes bearing a net negative charge without necessitating additional nanocarrier modifications which can affect the stability of nanomaterials $[8,25]$.

The efficacy of a chemotherapeutic drug and the side effects greatly depend on their release profile up to a certain extent [30]. There were several reports on the disintegration of LDH layers specifically in the acidic promoting drug release at the tumor microenvironment [31-33]. Thus, the release behavior of DOX from CuAl-LDH@DOX and CuAlLDH@DOX@PDA samples was evaluated in phosphate-buffered saline at two different pHs, viz 7.4 and 5.0, to simulate normal and tumor microenvironments (Figure 3a) [34]. In the traditional drug delivery methods, there is a rapid increase and decrease in the drug levels in the blood, which necessitates the administration of drugs at multiple-time intervals. In contrast, the extended drug delivery methods maintain a constant level of the drug for prolonged periods, which can drastically reduce the dose and frequency of drug intake, thereby alleviating the associated side effects. Nevertheless, one of the key challenges in extended drug delivery is the initial burst release of the drug in the early phases. In CuAl-LDH@DOX samples, the initial drug release was rapid (50\% within the first $5 \mathrm{~h}$ ) whereas, PDA coating over the surface of LDH allowed the extended-release of DOX with about $21 \%$ DOX release within the first $10 \mathrm{~h}$. In both CuAl-LDH@DOX and CuAl-LDH@DOX@PDA samples, there is a significant increase of drug release in acidic $\mathrm{pH}$ values when compared to basic $\mathrm{pH}$ due to the degradation of LDH layers, thereby increasing DOX release.

(a)

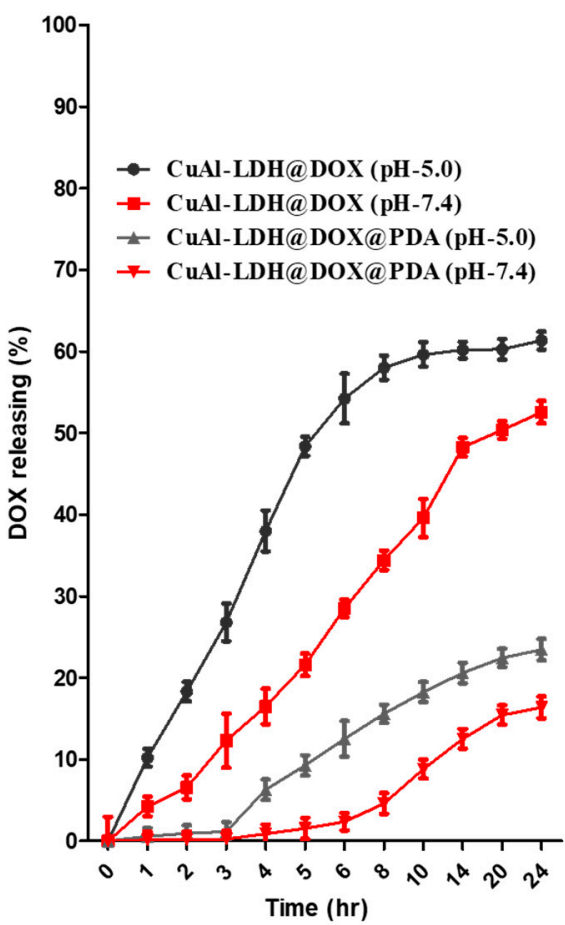

(b)

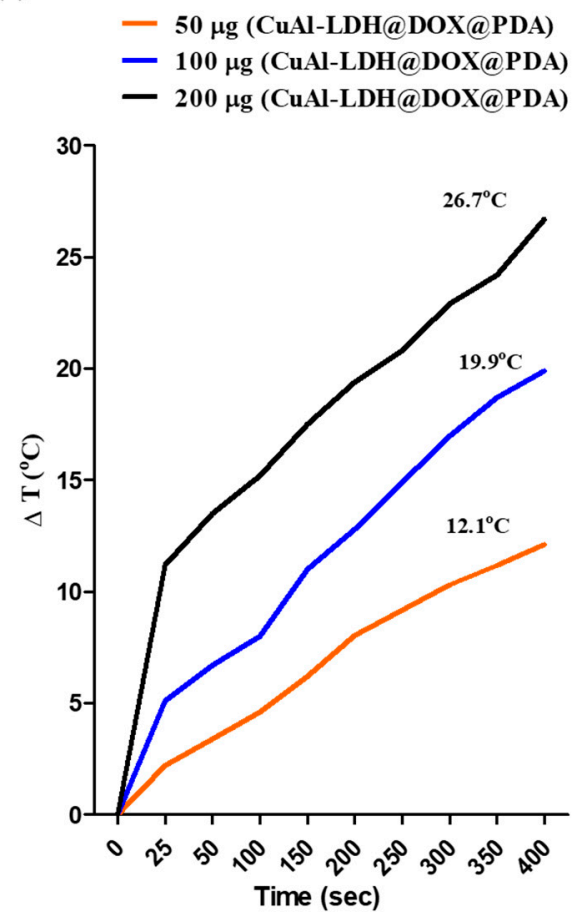

Figure 3. (a) DOX releasing from CuAl-LDH@DOX and CuAl-LDH@DOX@PDA over time at various PBS solutions of pH 5.0 and 7.4 (at $\left.37^{\circ} \mathrm{C}\right)$. (b) Temperature increasing profiles of CuAl-LDH@DOX@PDA under 808 nm laser irradiation over time $(50,100$, and $200 \mu \mathrm{g} / \mathrm{mL})$. 


\subsection{Photothermal Performance of CuAl-LDH@DOX@PDA}

In recent years, there were several reports on the ability of PDA to convert NIR light to heat for the destruction of tumors (Figure 3b) [34-36]. Therefore, we sought to investigate the photothermal properties of PDA. The photothermal properties of $\mathrm{CuAl}$ LDH@DOX@PDA are evaluated by heating the NC dispersions at different concentrations $(50,100$, and $250 \mu \mathrm{g} / \mathrm{mL}$ under NIR irradiation $(810 \mathrm{~nm})$ with a time interval between $25-400 \mathrm{~s}$. With the increase in time, there is a significant increase in temperature in a dosedependent manner. Our results provide strong evidence that photothermal performance can be controlled by varying the concentration of NC according to necessity.

\subsection{Cellular Uptake and Intracellular Distribution of NC}

To determine the endocytosis of CuAl-LDH, a co-localization analysis was performed in A549 cells using FITC conjugated LDH. The results indicated a time-dependent increase in cellular uptake. After $6 \mathrm{~h}$ of incubation, there is an increase in green fluorescence at the cytoplasm indicating successful endocytosis of LDH (Figure 4A) [25]. To investigate the uptake of NC intercalated DOX into the cells, A549 cells were incubated with CuAl-LDH@DOX@PDA samples for $24 \mathrm{~h}$ before taking fluorescence images. As shown in Figure 4B, A549 cells incubated with CuAl-LDH@DOX@PDA showed a strong red fluorescence, indicating a high uptake of NC through endocytosis which is in agreement with previous studies on LDH@DOX uptake $[29,37]$.

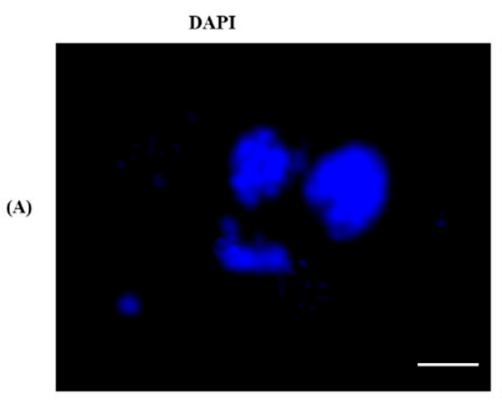

DAPI

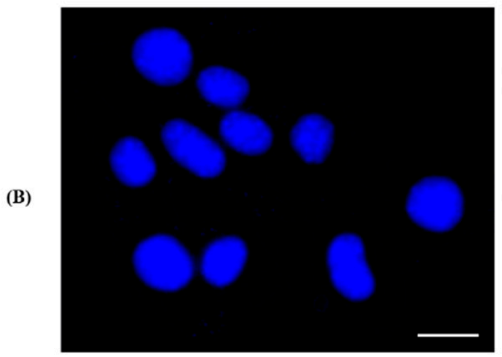

FITC

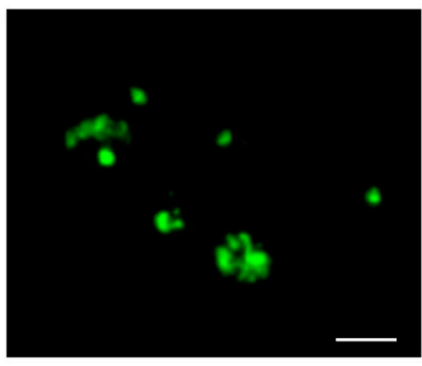

Dox

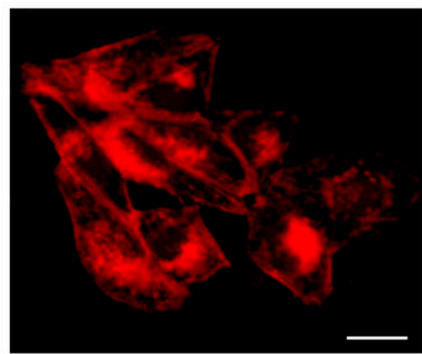

Merge

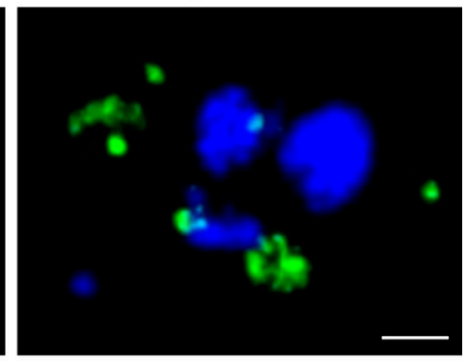

Merge

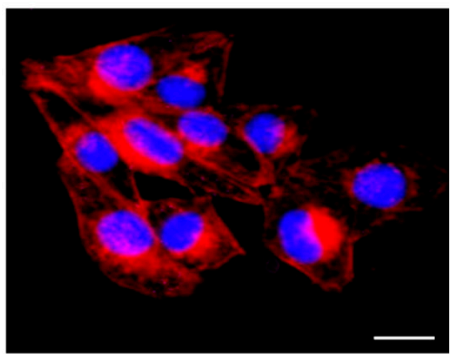

Figure 4. (A) Fluorescence microscopic images of CuAl-LDH $(100 \mu \mathrm{g} / \mathrm{mL})$ cellular uptake with the treatment of FITC labeled CuAl-LDH $(100 \mu \mathrm{g} / \mathrm{mL})$, and (B) intracellular DOX uptake in A549 cells treated with CuAl-LDH@DOX@PDA (20× magnification), (Scale bar $100 \mu \mathrm{m})$.

\subsection{In Vitro Cell Viability and ROS Production}

The in vitro therapeutic efficacy of $\mathrm{NC}$ was verified using methyl thiazolyl tetrazolium (MTT) assay in cells treated with DOX, CuAl-LDH@DOX, and CuAl-LDH@DOX@PDA with and without light at different drug concentrations. As seen in Figure 5. CuAlLDH@DOX@PDA displayed better cytotoxic effects than DOX and CuAl-LDH@DOX at all the tested concentrations. In addition, light irradiation significantly reduced the number of viable cells (IC $5012.5 \mathrm{ng} / \mathrm{mL}$ for DOX, CuAl-LDH@DOX, and CuAl-LDH@DOX (-L), $6.25 \mathrm{ng} / \mathrm{mL}$ for CuAl-LDH@DOX (+L)) in CuAl-LDH@DOX@PDA treated samples conforming to chemo-photothermal synergistic properties of the NC similar to previous findings. 


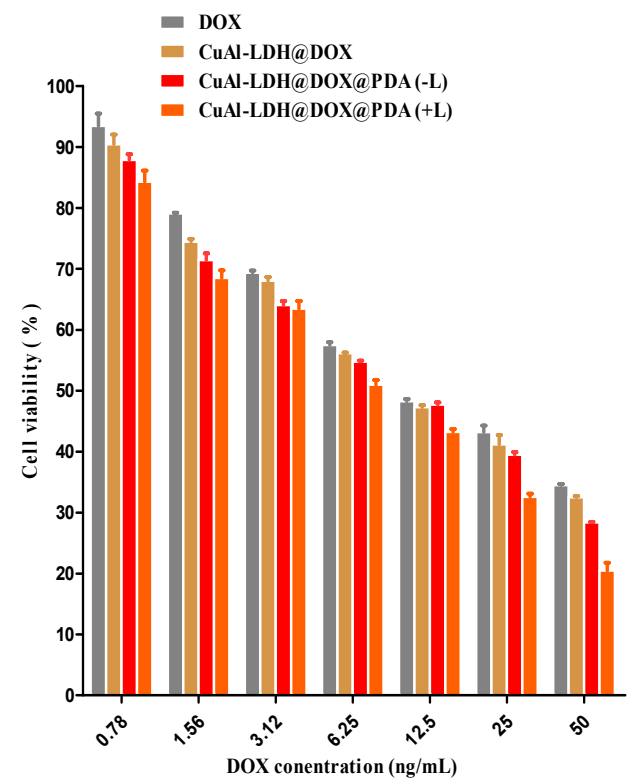

Figure 5. In vitro cell viability studies, dose-dependent cell viability of A549 cells incubated with DOX,CuAl-LDH@DOX,CuAl-LDH@DOX@PDA, and CuAl-LDH@DOX@PDA (-L) for 24 h and CuAl-LDH@DOX@PDA (+L) treated groups were irradiation light source for $5 \mathrm{~min}$ after $24 \mathrm{~h}$ treatment (DOX equivalent dose).

Intracellular ROS levels were determined in A549 cells using ROS-sensitive fluorescent dye, $2^{\prime}, 7^{\prime}$-dichlorodihydrofluorescein diacetate (DCFH-DA). DCFH-DA emits a green, fluorescent signal upon reacting with ${ }^{1} \mathrm{O}_{2}$ oxygen species. As shown in Figure 6, cells without NC (negative control) incubation exhibited a weak fluorescence signal, while cells treated with $\mathrm{H}_{2} \mathrm{O}_{2}$ (positive control) displayed a strong fluorescent signal confirming the presence of ROS.CuAl-LDH@DOX and CuAl-LDH@DOX@PDA samples displayed similar fluorescent signals in the absence of light, however, NIR irradiation exhibited a significant increase in ROS levels in CuAl-LDH@DOX@PDA samples, an additional confirmatory test that revealed CDT/PTT synergistic properties of the NC.

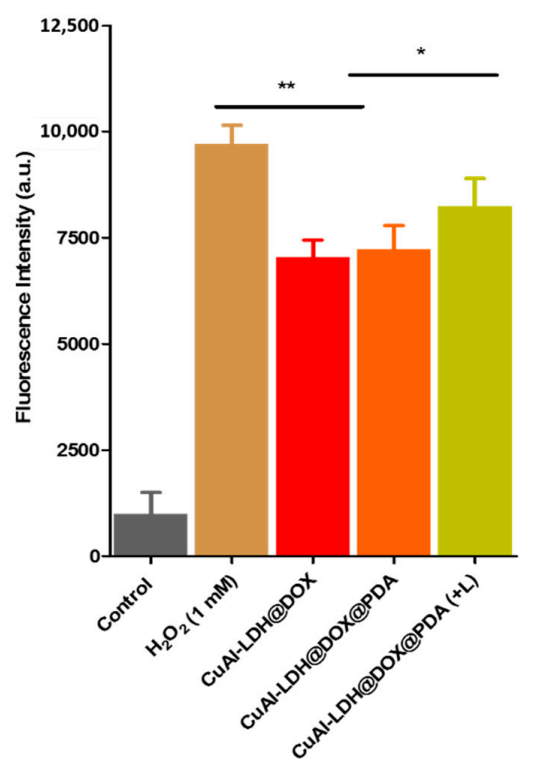

Figure 6. Intracellular ROS production in A549 cells treated with NCs with and without light irradiation by DCFDA assay. The fluorescence signal was detected by a microplate reader $\left(\kappa_{\mathrm{ex}}=488 \mathrm{~nm}\right.$, $\kappa_{\mathrm{em}}=535 \mathrm{~nm}$ ). 


\subsection{Effects of NC on Reduced GSH Levels}

Within the cells, glutathione exists in a reduced monomeric form (GSH) or an oxidized dimeric form (GSSG). Usually, most of the cellular glutathione exists in the reduced state (95\%) [38] and a decrease in intracellular GSH levels indicates utilization of GSH or development of GSH-adducts one assessment of cellular oxidative stress. We assessed the effects of CuAl-LDH, CuAl-LDH@DOX, and CuAl-LDH@DOX@PDA with and without light irradiation on reduced GSH levels (Figure 7). Treatment with CuAl-LDH, CuAl-LDH@DOX significantly reduced the GSH levels when compared to CuAl-LDH. Additionally, light irradiation significantly depleted GSH levels in CuAl-LDH@DOX@PDA due to the synergistic CDT/PTT properties.

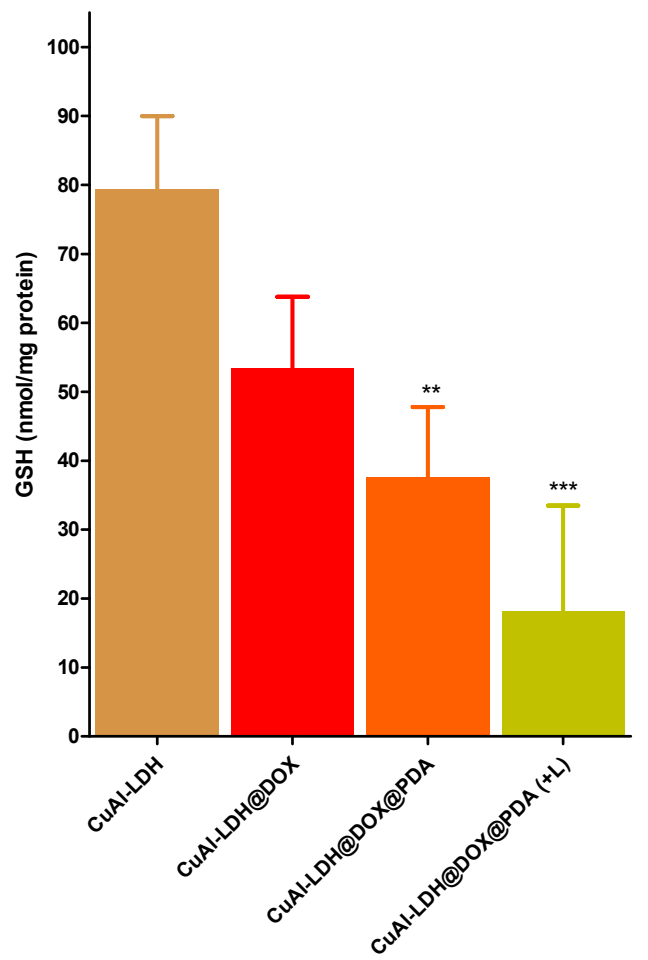

Figure 7. NCs inhibits GSH production in A549 cells. Different NCs inhibit GSH production in A549 cells compared with $0 \mu \mathrm{M}$ of the treatment group. ${ }^{* *}$ and ${ }^{* *}$ denotes the significant differences of CuAl-LDH@DOX@PDA,CuAl-LDH@DOX@PDA (+L) compared with $0 \mu \mathrm{M}$ of NC.

\section{Conclusions}

In summary, we established a multifunctional CuAl-LDH@DOX@PDA NC for combined chemo dynamic and photothermal therapy for the ablation of A549 cancer cells. NC's successfully delivered the DOX to the cancer cells efficiently in an acidic environment and participated in the CDT sequential reactions to enhance the ROS levels. In addition, efficient PTT therapy under specified light sources potentially enhanced the cancer therapy. Therefore, CuAl-LDH@DOX@PDA NC's can serve as a good platform for the selective eradication of cancer cells.

\section{Limitations and Future Perspectives}

Although LDH are efficient materials for therapeutic delivery with high biocompatibility, their inability to surface functionalize biomolecules limits their scope in designing particles with multiple properties. This can be overcome through the development of hybrid nanocarriers by a combination of LDH with other inorganic nanocarriers such as mesoporous silica, metal, and non-metal nanocarriers, etc., that can be effectively functionalized for multiple functions. 
Author Contributions: Conceptualization and methodology, software, formal analysis, writingoriginal draft preparation, validation, resources and funding, P.B.; validation, formal analysis, investigation, R.K.; data curation, supervision, resources, D.-Y.L.; supervision, editing, funding acquisition, J.S.; conceptualization, and methodology, software, formal analysis, writing-original draft preparation, validation, resources and funding, Y.K. All authors have read and agreed to the published version of the manuscript.

Funding: This research was supported by the National Research Foundation of Korea (NRF) funded by the Korea Government (2020R1A2C1012439), Republic of Korea.

Institutional Review Board Statement: We have not condcuted any experiments on animal or humans.

Informed Consent Statement: Not applicable.

Data Availability Statement: No supporting data presented in this study.

Acknowledgments: Authors are thankful to Shaik Gouse Peera, Department of Environmental Science and Engineering, Keimyung University, Republic of Korea for a constructive technical discussion.

Conflicts of Interest: The authors declare that they have no known competing financial interests or personal relationships that could have appeared to influence the work reported in this paper.

\section{References}

1. Sung, H.; Ferlay, J.; Siegel, R.L.; Laversanne, M.; Soerjomataram, I.; Jemal, A.; Bray, F. Global cancer statistics 2020: GLOBOCAN estimates of incidence and mortality worldwide for 36 cancers in 185 countries. CA Cancer J. Clin. 2021, 71, 209-249. [CrossRef]

2. Utku, N. New approaches to treat cancer-What they can and cannot do. Biotechnol. Healthc. 2011, 8, 25-27. [PubMed]

3. Zugazagoitia, J.; Guedes, C.; Ponce, S.; Ferrer, I.; Molina-Pinelo, S.; Paz-Ares, L. Current challenges in cancer treatment. Clin. Ther. 2016, 38, 1551-1566. [CrossRef] [PubMed]

4. Mokhtari, R.B.; Homayouni, T.S.; Baluch, N.; Morgatskaya, E.; Kumar, S.; Das, B.; Yeger, H. Combination therapy in combating cancer. Oncotarget 2017, 8, 38022-38043. [CrossRef] [PubMed]

5. Xiao, S.; Lu, Y.; Feng, M.; Dong, M.; Cao, Z.; Zhang, X.; Chen, Y.; Liu, J. Multifunctional FeS 2 theranostic nanoparticles for photothermal-enhanced chemodynamic/photodynamic cancer therapy and photoacoustic imaging. Chem. Eng. J. 2020, 396, 125294. [CrossRef]

6. Nie, X.; Xia, L.; Wang, H.-L.; Chen, G.; Wu, B.; Zeng, T.-Y.; Hong, C.-Y.; Wang, L.-H.; You, Y.-Z. Photothermal therapy nanomaterials boosting transformation of $\mathrm{Fe}(\mathrm{III})$ into Fe(II) in tumor cells for highly improving chemodynamic therapy. ACS Appl. Mater. Interfaces 2019, 11, 31735-31742. [CrossRef]

7. Kuthati, Y.; Sung, P.J.; Weng, C.F.; Mou, C.Y.; Lee, C.H. Functionalization of mesoporous silica nanoparticles for targeting, biocompatibility, combined cancer therapies and theragnosis. J. Nanosci. Nanotechnol. 2013, 13, 2399-2430. [CrossRef]

8. Kuthati, Y.; Kankala, R.K.; Lee, C.-H. Layered double hydroxide nanoparticles for biomedical applications: Current status and recent prospects. Appl. Clay Sci. 2015, 112-113, 100-116. [CrossRef]

9. Calixto, G.M.F.; Bernegossi, J.; De Freitas, L.M.; Fontana, C.R.; Chorilli, M. Nanotechnology-based drug delivery systems for photodynamic therapy of cancer: A review. Molecules 2016, 21, 342. [CrossRef]

10. Aflori, M. Smart nanomaterials for biomedical applications-A review. Nanomaterials 2021, 11, 396. [CrossRef]

11. Cunha, V.R.R.; de Souza, R.B.; da Fonseca Martins, A.M.C.R.P.; Koh, I.H.J.; Constantino, V.R.L. Accessing the biocompatibility of layered double hydroxide by intramuscular implantation: Histological and microcirculation evaluation. Sci. Rep. 2016, 6, 30547. [CrossRef]

12. Choi, S.J.; Choy, J.H. Layered double hydroxide nanoparticles as target-specific delivery carriers: Uptake mechanism and toxicity. Nanomedicine 2011, 6, 803-814. [CrossRef] [PubMed]

13. Qin, L.; Xue, M.; Wang, W.; Zhu, R.; Wang, S.; Sun, J.; Zhang, R.; Sun, X. The in vitro and in vivo anti-tumor effect of layered double hydroxides nanoparticles as delivery for podophyllotoxin. Int. J. Pharm. 2010, 388, 223-230. [CrossRef]

14. Yan, L.; Gonca, S.; Zhu, G.; Zhang, W.; Chen, X. Layered double hydroxide nanostructures and nanocomposites for biomedical applications. J. Mater. Chem. B 2019, 7, 5583-5601. [CrossRef] [PubMed]

15. Lu, J.; Guo, Z.; Che, S.; Gao, F.; Gu, Z.; Xu, J.; Chi, Y.; Xu, W.; Zhang, J.; Takuya, N.; et al. Dihydroartemisinin loaded layered double hydroxide nanocomposites for tumor specific photothermal-chemodynamic therapy. J. Mater. Chem. B 2020, 8, 11082-11089. [CrossRef] [PubMed]

16. Sun, L.; Wang, J.; Liu, J.; Li, L.; Xu, Z.P. Creating structural defects of drug-free copper-containing layered double hydroxide nanoparticles to synergize photothermal/photodynamic/chemodynamic cancer therapy. Small Struct. 2021, 2, 2000112. [CrossRef]

17. Jia, T.; Wang, Z.; Sun, Q.; Dong, S.; Xu, J.; Zhang, F.; Feng, L.; He, F.; Yang, D.; Yang, P.; et al. Intelligent Fe-Mn layered double hydroxides nanosheets anchored with upconversion nanoparticles for oxygen-elevated synergetic therapy and bioimaging. Small 2020, 16, e2001343. [CrossRef]

18. Ruan, Y.; Jia, X.; Wang, C.; Zhen, W.; Jiang, X. Mn-Fe layered double hydroxide nanosheets: A new photothermal nanocarrier for $\mathrm{O}_{2}$-evolving phototherapy. Chem. Commun. 2018, 54, 11729-11732. [CrossRef] 
19. Liu, J.; Sun, L.; Li, L.; Zhang, R.; Xu, Z.P. Synergistic cancer photochemotherapy via layered double hydroxide-based trimodal nanomedicine at very low therapeutic doses. ACS Appl. Mater. Interfaces 2021, 13, 7115-7126. [CrossRef]

20. Wang, Z.; Fu, L.; Zhu, Y.; Wang, S.; Shen, G.; Jin, L.; Liang, R. Chemodynamic/photothermal synergistic therapy based on ce-doped Cu-Al layered double hydroxides. J. Mater. Chem. B 2021, 9, 710-718. [CrossRef]

21. Wen, J.; Yang, K.; Huang, J.; Sun, S. Recent advances in LDH-based nanosystems for cancer therapy. Mater. Des. 2021, $198,109298$. [CrossRef]

22. Wijitwongwan, R.; Intasa-ard, S.; Ogawa, M. Preparation of layered double hydroxides toward precisely designed hierarchical organization. ChemEngineering 2019, 3, 68.

23. Feng, L.; Dong, Z.; Tao, D.; Zhang, Y.; Liu, Z. The acidic tumor microenvironment: A target for smart cancer nano-theranostics. Natl. Sci. Rev. 2017, 5, 269-286. [CrossRef]

24. Ranji-Burachaloo, H.; Gurr, P.A.; Dunstan, D.E.; Qiao, G.G. Cancer treatment through nanoparticle-facilitated fenton reaction. ACS Nano 2018, 12, 11819-11837. [CrossRef] [PubMed]

25. Oh, J.M.; Choi, S.J.; Kim, S.T.; Choy, J.H. Cellular uptake mechanism of an inorganic nanovehicle and its drug conjugates: Enhanced efficacy due to clathrin-mediated endocytosis. Bioconjug. Chem. 2006, 17, 1411-1417. [CrossRef]

26. Mei, X.; Xu, S.; Hu, T.; Peng, L.; Gao, R.; Liang, R.; Wei, M.; Evans, D.G.; Duan, X. Layered double hydroxide monolayers for controlled loading and targeted delivery of anticancer drugs. Nano Res. 2017, 11, 195-205. [CrossRef]

27. Dreyer, D.R.; Miller, D.J.; Freeman, B.D.; Paul, D.R.; Bielawski, C.W. Elucidating the structure of poly(dopamine). Langmuir 2012, 28, 6428-6435. [CrossRef]

28. Kuthati, Y.; Busa, P.; Tummala, S.; Rao, V.N.; Davuluri, V.N.G.; Ho, Y.-P.; Wong, C.-S. Mesoporous polydopamine nanoparticles attenuate morphine tolerance in neuropathic pain rats by inhibition of oxidative stress and restoration of the endogenous antioxidant system. Antioxidants 2021, 10, 195. [CrossRef]

29. Hakeem, A.; Zhan, G.; Xu, Q.; Yong, T.; Yang, X.; Gan, L. Facile synthesis of pH-responsive doxorubicin-loaded layered double hydroxide for efficient cancer therapy. J. Mater. Chem. B 2018, 6, 5768-5774. [CrossRef]

30. Li, Y.; Xiao, K.; Zhu, W.; Deng, W.; Lam, K.S. Stimuli-responsive cross-linked micelles for on-demand drug delivery against cancers. Adv. Drug Deliv. Rev. 2014, 66, 58-73. [CrossRef]

31. Mishra, D.; Banerjee, D. Lactate dehydrogenases as metabolic links between tumor and stroma in the tumor microenvironment. Cancers 2019, 11, 750. [CrossRef]

32. Xu, T.; Liu, J.; Sun, L.; Zhang, R.; Xu, Z.P.; Sun, Q. Enhancing tumor accumulation and cellular uptake of layered double hydroxide nanoparticles by coating/detaching pH-triggered charge-convertible polymers. ACS Omega 2021, 6, 3822-3830. [CrossRef] [PubMed]

33. Huang, G.; Zhang, K.L.; Chen, S.; Li, S.H.; Wang, L.L.; Wang, L.P.; Liu, R.; Gao, J.; Yang, H.H. Manganese-iron layered double hydroxide: A theranostic nanoplatform with $\mathrm{pH}$-responsive MRI contrast enhancement and drug release. J. Mater. Chem. B 2017, 5, 3629-3633. [CrossRef] [PubMed]

34. Paliwal, S.R.; Paliwal, R.; Agrawal, G.P.; Vyas, S.P. Hyaluronic acid modified pH-sensitive liposomes for targeted intracellular delivery of doxorubicin. J. Liposome Res. 2016, 26, 276-287. [CrossRef]

35. Xing, Y.; Zhang, J.; Chen, F.; Liu, J.; Cai, K. Mesoporous polydopamine nanoparticles with co-delivery function for overcoming multidrug resistance via synergistic chemo-photothermal therapy. Nanoscale 2017, 9, 8781-8790. [CrossRef]

36. Lei, W.; Sun, C.; Jiang, T.; Gao, Y.; Yang, Y.; Zhao, Q.; Wang, S. Polydopamine-coated mesoporous silica nanoparticles for multi-responsive drug delivery and combined chemo-photothermal therapy. Mater. Sci. Eng. C 2019, 105, 110103. [CrossRef]

37. Al-Ali, S.H.H.; Al-Qubaisi, M.; Hussein, M.Z.; Ismail, M.; Bullo, S. Hippuric acid nanocomposite enhances doxorubicin and oxaliplatin-induced cytotoxicity in MDA-MB231, MCF-7 and Caco2 cell lines. Drug Des. Dev. Ther. 2013, 7, 25-31. [CrossRef]

38. Wataha, J.C.; Lewis, J.B.; Lockwood, P.E.; Rakich, D.R. Effect of dental metal ions on glutathione levels in THP-1 human monocytes. J. Oral Rehabil. 2000, 27, 508-516. [CrossRef] 